\title{
EDITORIAL \\ Improvements to the manufacturing process for generation of Schwann cells: from laboratory to clinical translation
}

\author{
Mayank Kaushal, MD, MBA, and Shekar N. Kurpad, MD, PhD \\ Department of Neurological Surgery, The Medical College of Wisconsin, Milwaukee, Wisconsin
}

$\mathrm{I}$ $\mathrm{N}$ their article, Khan and associates provide a comprehensive overview of the manufacturing protocol for the production of clinical-grade autologous Schwann cells (SCs), which is compliant with the Current Good Manufacturing Practices (CGMPs). ${ }^{1}$ The methodology for obtaining an appropriate sample of SCs for the purpose of transplantation involves a number of complex steps, which are articulated in a clear manner.

In the process of documenting their observations, the authors have brought up a number of noteworthy points that deserve recognition. The finding of an increase in cell number at each passage that is independent of the length of the harvested nerve is evidence that the protocol performs consistently. The fact that a cell purity percentage in excess of $90 \%$ was seen with most of the donor nerve samples indicates the importance of a meticulous approach towards the removal of fascicles from the epineurium. The analysis mentions the interval of time during which the clinical product remains stable after preparation, which should serve as a guide when designing future transplantation trials. Furthermore, a tabulated description of the various tests performed on the SCs with each of the tests performed at multiple steps during the creation of the clinical product serves to enhance the credibility of the final clinical product transplanted in the patients. A testament to the strength of the protocol is the fact that cultures of SCs developed from both injured and uninjured peripheral nerves were similar when compared for viability and purity during each passage. Another feature of the manufacturing process that merits attention is that cryopreservation did not result in any negative impact on characteristics of the SC cultures, such cell viability, cell expansion, and genetic stability. This is a promising development, with the potential for making transplantation of SCs an option in a greater number of patients suffering from chronic nervous system injury.

Drawing on the lessons learned over the course of their extensive exposure to cell transplantation, the authors successfully highlight the clinical translation potential of SCs in a diverse cohort comprising patients with subacute spinal cord injury (SCI), chronic SCI, and peripheral nerve injuries. The results prove the feasibility of production of patient-specific SCs in large quantities from a relatively small length of peripheral nerve obtained from the patient in question. The work assumes even more significance as it comes with optimizations made to manufacturing processes that combine reductions in time with consistent cell yields.

The authors are to be congratulated for developing this excellent technique, which will likely be applicable to a wide range of neurological maladies.

https://thejns.org/doi/abs/10.3171/2021.1.SPINE202065

\section{References}

1. Khan A, Diaz A, Brooks AE, et al. Scalable culture techniques to generate large numbers of purified human Schwann cells for clinical trials in human spinal cord and peripheral nerve injuries. J Neurosurg Spine. Published online September 3, 2021. doi:10.3171/2020.11.SPINE201433

\section{Disclosures}

The authors report no conflict of interest.

\section{Correspondence}

Shekar N. Kurpad: skurpad@mcw.edu.

INCLUDE WHEN CITING

Published online September 3, 2021; DOI: 10.3171/2021.1.SPINE202065.

\section{Response}

\section{Aisha Khan, MSc, MBA, ${ }^{1,3}$ and Allan D. Levi, MD, $\mathrm{PhD}^{1,2}$ \\ ${ }^{1}$ The Miami Project to Cure Paralysis, ${ }^{2}$ Department of Neurological Surgery, and ${ }^{3}$ Interdisciplinary Stem Cell Institute, University of Miami Miller School of Medicine, Miami, Florida}

We thank the authors for their comments regarding cell number, cell purity, our meticulous approach to prepar- 
ing the cells from the donor nerve using a CGMP facility, the product assessment, the ability of cells to be cryopreserved and reprocessed, and the overall optimization of the techniques over time. The work really summates a 30year process of investigation into the biology of human SCs and their potential use clinically as a regenerative product for nervous system injury. We are deeply indebted to all the scientists and clinicians who have contributed to this work, first starting with Dr. Richard Bunge who, unfortunately, passed away some 25 years ago and is dearly missed by all.

\section{INCLUDE WHEN CITING}

Published online September 3, 2021; DOI: 10.3171/2021.2.SPINE2193. 